\title{
FEOFORBÍDEO (ETOXI-PURPURINA-18) ISOLADO DE Gossypium mustelinum (MALVACEAE)
}

\section{Tania Maria Sarmento Silva* e Celso Amorim Camara}

Departamento de Química, Universidade Federal Rural de Pernambuco, 52171-900 Recife - PE, Brasil

José Maria Barbosa-Filho

Laboratório de Tecnologia Farmacêutica, Universidade Federal da Paraíba, CP 5009, 58051-970 João Pessoa - PB, Brasil

Ana Maria Giulietti

Departamento de Ciências Biológicas, Universidade Estadual de Feira de Santana, 44031-640 Feira de Santana - BA, Brasil

Recebido em 6/3/09; aceito em 28/8/09; publicado na web em 11/1/10

ETHYL ESTER PURPURIN-18 FROM Gossypium mustelinum (MALVACEAE). The phaeophorbide ethyl ester named Purpurin-18 and the flavonoids quercetin and kaempferol were obtained by chromatographic procedures from the chloroform fraction of aerial parts of Gossypium mustelinum. The structure of these compound was determined by NMR, IR and mass spectra data analysis. This is the first occurrence of this compound in Angiosperm.

Keywords: Gossypium mustelinum; phaeophorbide; ethyl ester purpurin-18.

\section{INTRODUÇÃO}

O gênero Gossypium L. (Malvaceae) apresenta cerca de 50 espécies (45 espécies diploides e cinco alelotetraploides), ${ }^{1}$ distribuídas, principalmente, na África, Austrália, Peru, México, Arábia e Brasil. O Brasil é considerado um importante centro de distribuição de três espécies (G. barbadense L., G. barbadense var. brasiliense Hutch. e G. hirsutum var. Marie galante Hutch.) e o centro de origem da espécie Gossypium mustelinum, conhecido popularmente como "algodão bravo", que é uma espécie de algodoeiro selvagem, alelotetraploide, arbórea, endêmica do semiárido, no nordeste do Brasil. ${ }^{2}$ Em nosso trabalho anterior com a fração clorofórmica das partes aéreas desta espécie foi mostrado o isolamento de três feofitinas: faeofitina $\mathrm{B}$, $17^{3}$-etoxifaeoforbídeo A e faeoforbídeo A. ${ }^{3}$

Substâncias naturais que estão relacionadas com a clorofila são relativamente raras na natureza, devido, provavelmente ao alto potencial fotodinâmico destes pigmentos. ${ }^{4} \mathrm{~A}$ diversidade estrutural das feofitinas/feoforbídeos e as atividades biológicas que apresentam têm despertado muito interesse, principalmente na terapia fotodinâmica do câncer, sendo um novo método de tratamento com drogas menos tóxicas (sensibilizadores) que utiliza a luz visível e do infravermelho próximo (VIS/NIR) combinadas para gerar espécies citotóxicas derivadas de oxigênio (ROS) em um lugar selecionado do tratamento.

Dando continuidade ao estudo químico de G. mustelinum é descrito neste trabalho o isolamento e a completa determinação estrutural da etoxi-purpurina-18.

\section{RESULTADOS E DISCUSSÃO}

O extrato etanólico das partes aéreas de G. mustelinum foi submetido à partição com solventes orgânicos. ${ }^{3} \mathrm{~A}$ fração clorofórmica foi submetida à cromatografia com Sephadex LH-20 e sílica gel e forneceu o feoforbídeo $\mathbf{1}$ e os flavonoides $\mathbf{2}$ (quercetina) e $\mathbf{3}$ (kanferol). A substância $\mathbf{1}$ foi isolada como um sólido amorfo preto azulado. A análise obtida através de CL-EM-ESI (ionização por electrospray) no modo positivo mostrou o pico do íon molecular em $\mathrm{m} / \mathrm{z}$,593,42 $[\mathrm{M}+\mathrm{H}]^{+}$. A fórmula molecular $\mathrm{C}_{35} \mathrm{H}_{36} \mathrm{~N}_{4} \mathrm{O}_{4}$ foi deduzida pelos dados obtidos da CL-EM, RMN ${ }^{1} \mathrm{He} \mathrm{e}^{13} \mathrm{C}$-APT (Tabela 1) correspondendo a

\footnotetext{
*e-mail: taniasarmento@dq.ufrpe.br
}

20 graus de insaturação. O espectro de IV $(\mathrm{KBr})$ apresentou absorções em $3433(\mathrm{NH}), 1723(\mathrm{C}=\mathrm{O}), 1631(\mathrm{C}=\mathrm{C}) \mathrm{cm}^{-1}$. A cor preta azulada característica indicou a presença de um grande cromóforo que pode estar relacionado com a clorofila ou seus derivados.

$\mathrm{O}$ espectro de $\mathrm{RMN}{ }^{1} \mathrm{H}$ mostrou absorções características para a substância do tipo feoforbídeo ou seus derivados ${ }^{6}$ pela presença de três grupos metilas ligadas a anel aromático $\left(\delta_{\mathrm{H}} 3,68 ; 3,32\right.$ e 3,08$)$, três sinais de hidrogênios olefínicos em $\delta_{\mathrm{H}} 9,44 ; 9,27$ e 8,54 , e um grupo vinila $\left(\delta_{\mathrm{H}}\right.$ 6,$25 ; 5,15$ e 7,83) com um padrão característico para metileno, além da ausência da cadeia fitila, uma vez que o envelope característico de sinais na região alifática de grupos metilenos das feofitinas se encontra ausente. ${ }^{6}$

O espectro de APT junto com HMQC mostrou a presença de seis metilas, cinco grupos metilenos (quatro alifáticos e um olefínico), seis metínicos (dois alifáticos e quatro olefínicos) e dezoito carbonos quaternários (15 olefínicos e três carbonilas). Os dados de RMN e EM (ESI), associados com os graus de insaturação permitiram estabelecer a estrutura como um anidrido cíclico conjugado de seis membros, supostamente presente entre os carbonos $\mathrm{C}-13^{1}-\mathrm{C}-13^{2}$ no anel $\mathrm{E}$, com dois valores de carbonila em $\delta_{\mathrm{C}} 164,17$ e 159,36 , além de um grupo etoxila $\left(\delta_{\mathrm{C}} 60,40\right.$ e 14,2$)$, provavelmente no lugar da cadeia fitila.

O completo assinalamento da estrutura do núcleo porfirina da feoforbida 1 foi realizada através dos assinalamentos a longa distância ${ }^{2,3} J_{\mathrm{CH}}$ das metilas aromáticas $3 \mathrm{H}-12^{1}, 3 \mathrm{H}-2^{1}$ e $3 \mathrm{H}-7^{1}$ e uma alifática $3 \mathrm{H}-18^{1}$, que mostraram correlações entre os quatro anéis pirrólicos (Figura 1). O sinal da metila $2^{1}\left(\delta_{\mathrm{H}} 3,32\right)$ mostrou correlação com os carbonos C-1, C-2 e C-3 (anel A), a metila $7^{1}$ correlacionou com C-7 e C-8 (anel B). No anel $\mathrm{C}$ verificou-se a correlação da metila $12^{1}$ com os carbonos C-11, C-12 e C-13 e, finalmente, a metila $18^{1}$ correlacionou-se com C-17, C-18 e C-19 (anel D). A correlação ${ }^{2} J_{\mathrm{CH}}$ entre os hidrogênios $\delta_{\mathrm{H}} 17^{4}(4,03), 17^{2}-\mathrm{B}\left(\delta_{\mathrm{H}}\right.$ $2,37)$ e $17^{2}-\mathrm{A}(2,66)$ com a carbonila em $\mathrm{C}-17^{3}\left(\delta_{\mathrm{C}} 173,25\right)$ confirmou a presença do grupo etoxi ligado à carboxila terminal em C-17.

A configuração relativa em C-17 e C-18 foi determinada como sendo trans, devido à interação espacial observada através do espectro de NOESY do H-17 $\left(\delta_{\mathrm{H}}, 13\right)$ com a metila $3 \mathrm{H}-18^{1}\left(\delta_{\mathrm{H}}, 72\right)$, e a comparação com os dados da literatura aponta que a configuração absoluta mais provável seria $17-S$ e $18-S,{ }^{6}$ (Figura 1). A análise de todos os dados permitiu identificar 1 como sendo a etoxi-purpurina-18. Esta substância está sendo isolada pela primeira vez como uma substância natural. Existe somente um relato na literatura da existência deste composto como produto de síntese. ${ }^{7}$ 


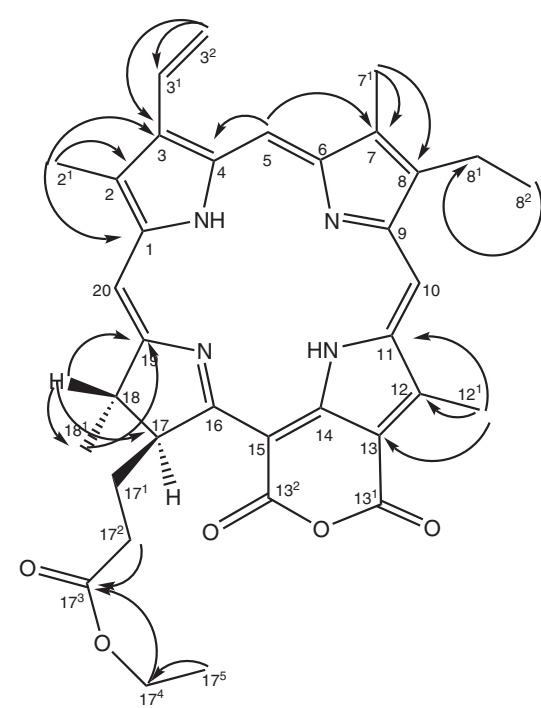

Figura 1. Estrutura do composto 1 e principais correlações observadas no espectro de $H M B C$

As purpurinas-18 ocorrem naturalmente somente em organismos marinhos e em sedimentos. ${ }^{8-12}$ São biossintetizadas nos próprios organismos marinhos, apresentando atividade antioxidante. ${ }^{8}$ Seus derivados são alvo de muito interesse devido à sua utilização como anticancerígeno. ${ }^{13-15}$

O isolamento de mais um feoforbídeo de G. mustelinum nos conduz à suposição de que esta é uma substância que ocorre naturalmente, e não um artefato produzido durante o processo de isolamento. De acordo com o estudo realizado anteriormente, ${ }^{3}$ é possível que estes pigmentos ocorram naturalmente na planta. Observando-se a espécie vegetal in natura alguns "pontos" de forte pigmentação escura ("pretos") são visíveis em toda parte vegetal. A hipótese de que estes pontos sejam regiões de concentração de pigmentos desta natureza e qual é a sua função precisa de maior fundamentação. Assim, é possível que os pigmentos presentes em G. mustelinum ocorram naturalmente, mas a hipótese de que sejam produtos de transesterificação e/ou alomerização, formados durante o processo de extração/isolamento, não pode ser inteiramente descartada. Outras espécies vegetais coletadas na região semiárida, como Anisacanthus brasiliensis ${ }^{16}$ e Sida galheirensis, ${ }^{17}$ também apresentaram a presença de feofitinas.

\section{PARTE EXPERIMENTAL}

\section{Procedimentos experimentais gerais}

As medidas de ponto de fusão foram efetuadas em um aparelho munido de placa de Koefler eletricamente alimentada e não foram corrigidas. Os espectros de infravermelho foram feitos em pastilhas de $\mathrm{KBr}$ em um espectrômetro Bomem/MB-102. Os espectros de Ressonância Magnética Nuclear, ${ }^{1} \mathrm{H}$ e ${ }^{13} \mathrm{C}$ (1D e 2D) foram registrados em espectrômetros Bruker DRX-500 $\left({ }^{1} \mathrm{H}: 500 \mathrm{MHz} ;{ }^{13} \mathrm{C}\right.$ : $\left.125 \mathrm{MHz}\right)$ (CENAUREMN-UFC) e Bruker AC $200\left({ }^{1} \mathrm{H} 200 \mathrm{MHz}\right.$ e ${ }^{13} \mathrm{C}$ 50,3 $\mathrm{MHz}$ ) em $\mathrm{CDCl}_{3}$ utilizando DMSO-d $\mathrm{d}_{6}$ ou $\mathrm{CDCl}_{3}$ como solvente e TMS como referência interna. O CL-EM (LTF-UFPB) foi utilizado no modo electrospray positivo com um Quattro LC-Micromass (Waters). Silica gel 60 (Vetec) e Sephadex LH-20 (Sigma) foram utilizados para cromatografia em coluna. Usaram-se placas de sílica gel $60 \mathrm{PF}_{254}$ Merck para cromatografia em camada delgada analítica e como reveladores luz ultravioleta (254 e $366 \mathrm{~nm}$ ), soluções de $\mathrm{AlCl}_{3}-\mathrm{EtOH}$ (1\%) e ácido difenilbórico etanolamina-MeOH (NP, 1\%).
Tabela 1. Dados de RMN ${ }^{1} \mathrm{H}(200 \mathrm{MHz})$ e ${ }^{13} \mathrm{C}(50,3 \mathrm{MHz})$ para substância 1 obtidos por espectros de 2D (HSQC e HMBC, $500 \mathrm{MHz}$ ) em CDCl $\mathrm{CD}_{3}$. Deslocamentos químicos ( $\delta$, ppm) e constantes de acoplamento ( $J$ em Hz, parênteses)

\begin{tabular}{|c|c|c|c|c|}
\hline & $\delta_{c}$ & $\delta_{\mathrm{H}}$ & ${ }^{2} J_{\mathrm{CH}}$ & ${ }^{3} J_{\mathrm{CH}}$ \\
\hline \multicolumn{5}{|c|}{$\mathrm{C}$} \\
\hline 1 & 144,1 & & & $3 \mathrm{H}-2^{1}$ \\
\hline 2 & 131,8 & & $3 \mathrm{H}-2^{1}$ & \\
\hline $2^{1}$ & 11,9 & $3,32(\mathrm{~s})$ & & \\
\hline 3 & 137,7 & & & $3 \mathrm{H}-2^{1}, 2 \mathrm{H}-3^{2}$ \\
\hline $3^{1}$ & 128,3 & $7,83(18,0 ; 11,6)$ & $2 \mathrm{H}-3^{2}$ & \\
\hline $3^{2}$ & 123,7 & $\begin{array}{l}\text { (E) } 6,25(\mathrm{dl}, 18,0) \\
\text { (Z) } 6,25(\mathrm{dl}, 11,6)\end{array}$ & & \\
\hline 4 & 136,6 & & $\mathrm{H}-5$ & \\
\hline 5 & 103,1 & 9,27 (s) & & \\
\hline 6 & 156,3 & & & \\
\hline 7 & 136,6 & & $3 \mathrm{H}-7^{1}$ & H-5 \\
\hline $7^{1}$ & 11,0 & $3,08(\mathrm{~s})$ & & \\
\hline 8 & 145,9 & & & $3 \mathrm{H}-7^{1}$ \\
\hline $8^{1}$ & 19,3 & $3,52(\mathrm{~m})$ & $3 \mathrm{H}-8^{2}$ & \\
\hline $8^{2}$ & 17,4 & $1,59(\mathrm{t} ; 7,2)$ & & \\
\hline 9 & 150,1 & & & \\
\hline 10 & 107,6 & $9,44(\mathrm{~s})$ & & \\
\hline 11 & 131,5 & & & $3 \mathrm{H}-12^{1}$ \\
\hline 12 & 139,1 & & $3 \mathrm{H}-12^{1}$ & \\
\hline $12^{1}$ & 12,3 & $3,68(\mathrm{~s})$ & & \\
\hline 13 & 111,4 & & & $3 \mathrm{H}-12^{1}$ \\
\hline $13^{1 *}$ & 159,4 & & & \\
\hline $13^{2 *}$ & 164,8 & & & \\
\hline 14 & 139,9 & & & \\
\hline 15 & 92,6 & & & \\
\hline 16 & 177,6 & & & \\
\hline 17 & 54,9 & $5,13(\mathrm{dl})$ & & $3 \mathrm{H}-18^{1}$ \\
\hline $17^{1}$ & 31,7 & $\begin{array}{l}\text { A } 2,43(\mathrm{~m}) \\
\text { B } 1,98(\mathrm{~m})\end{array}$ & & H-18 \\
\hline $17^{2}$ & 32,7 & $\begin{array}{l}\text { A } 2,66(\mathrm{~m}) \\
\text { B } 2,37(\mathrm{~m})\end{array}$ & & \\
\hline $17^{3}$ & 173,3 & & $2 \mathrm{H}-17^{2}$ & $2 \mathrm{H}-17^{4}$ \\
\hline $17^{4}$ & 60,4 & $4,03(7,2)$ & $3 \mathrm{H}-17^{5}$ & \\
\hline $17^{5}$ & 14,1 & $1,14(\mathrm{t} ; 7,0)$ & & \\
\hline 18 & 49,2 & $4,37(\mathrm{dl})$ & $3 \mathrm{H}-18^{1}$ & \\
\hline $18^{1}$ & 23,8 & $1,72(\mathrm{~d} ; 7,2)$ & H-18 & \\
\hline 19 & 176,6 & & & $\mathrm{H}-18^{1}$ \\
\hline 20 & 94,9 & $8,54(\mathrm{~s})$ & & \\
\hline
\end{tabular}

*Estes valores podem ser trocados

\section{Material vegetal}

As partes aéreas de G. mustelinum foram coletadas em Anhaguera, Bahia, Brasil, em março de 2002 e identificada pela Profa. A. M. Giulietti. Uma exsicata (A. M. Giulietti, 2044) está depositada no Herbário da Universidade Estadual de Feira de Santana.

\section{Extração e isolamento}

O procedimento experimental é semelhante ao realizado em trabalho anterior. ${ }^{3} \mathrm{O}$ pó seco e pulverizado $(1,87 \mathrm{~kg})$ foi extraído com EtOH em temperatura ambiente. $\mathrm{O}$ extrato foi concentrado em rotaevaporador sob pressão reduzida fornecendo 205,6 g do extrato etanólico. Uma porção deste extrato (190,0 g) foi suspensa com $\mathrm{MeOH}_{2} \mathrm{H}_{2} \mathrm{O}(1: 1)$ e 
extraída com hexano e $\mathrm{CHCl}_{3}$. Os solventes foram removidos em rotaevaporador fornecendo os extratos hexânico $(89,5 \mathrm{~g})$ e clorofórmico $(3,8 \mathrm{~g})$. A fração clorofórmica $(2,1 \mathrm{~g})$ foi submetido à coluna com Sephadex $\mathrm{LH}-20^{\circledR}$ usando como eluentes $\mathrm{CHCl}_{3}: \mathrm{MeOH}$ (1:1) fornecendo 21 frações. As frações 15-16 (10,0 mg) e 19-21 (15,0 mg) desta coluna apresentaram cristais amarelos revelados intensamente em CCDA com o reagente NP para flavonoides. A análise dos espectros de $\mathrm{RMN}{ }^{1} \mathrm{H}$ e ${ }^{13} \mathrm{C}$ permitiu identificar as substâncias como sendo quercetina (2) e kanferol (3), respectivamente. As frações 3 e 4 foram ressubmetidas à cromatografia em coluna de sílica gel e forneceram a feofitina $\mathrm{B}^{3}$. As frações de 5 a 7 foram purificadas em Sephadex LH-20 mostrando a presença de um precipitado identificado como $17^{3}$-etoxifaeoforbídeo A. As frações de 6-14 foram ressubmetidas à cromatografia em coluna de sílica gel utilizando hexano e clorofórmio em ordem crescente de polaridade. Na fração 3 precipitou o metoxifaeoforbíde A. ${ }^{6} \mathrm{~A}$ fração 7 mostrou a presença de uma substância microcristalina de cor preto azulada $(35,0 \mathrm{mg})$, temperatura de fusão $145-146^{\circ} \mathrm{C}$, que foi submetida à analise espectroscópica (IV, CL-EM-ESI e RMN, incluindo 2D) e identificada como sendo a etoxipurpurina-18 (1).

Etoxipurpurina-18 (1). Sólido amorfo; pf 145-146 ${ }^{\circ} \mathrm{C}$; IV (KBr) $v_{\max } .3433,2934,1723,1631,1462 \mathrm{~cm}^{-1}$. RMN ${ }^{1} \mathrm{H}\left(\mathrm{CDCl}_{3}, 500 \mathrm{e}\right.$ $200 \mathrm{MHz}$ ) (Tabela 1), RMN ${ }^{13} \mathrm{C}\left(\mathrm{CDCl}_{3}, 50,3\right.$ e $\left.125 \mathrm{MHz}\right)$ (Tabela 1), EM-ESI (pos) $m / z 593,42[\mathrm{M}+\mathrm{H}]^{+}\left(\mathrm{C}_{35} \mathrm{H}_{36} \mathrm{~N}_{4} \mathrm{O}_{4}\right)$.

\section{AGRADECIMENTOS}

IMSEAR-CNPq, FACEPE pelos auxílios e bolsas concedidas. Ao Prof. E. de J. Oliveira (LTF-UFPB) pela realização dos espectros de massas. Ao Centro Nordestino de Aplicação e Uso de Ressonância Magnética Nuclear (CENAUREMN) - Programa de Pós-graduação em Química Orgânica, Departamento de Química Orgânica e Inorgânica, Centro de Ciências da Universidade Federal do Ceará, Fortaleza - Ceará, pelos espectros 1D e 2D de Ressonância Magnética Nuclear $\left(\mathrm{RMN}{ }^{1} \mathrm{H} \mathrm{e}{ }^{13} \mathrm{C}\right)$.

\section{REFERÊNCIAS}

1. Fryxell, P. A.; Rheedea 1992, 2, 108

2. Barroso, P. A. V.; Freire, E. C.; Amaral, J. A. B. do; Silva, M. T. ; Zonas de exclusão de algodoeiros transgênicos para preservação de espécies de Gossypium nativas ou naturalizadas, Embrapa Algodão: Campina Grande, 2005 (Embrapa Algodão, Comunicado técnico, 242).

3. Silva, T. M. S.; Camara, C. A.; Medeiros, F. D.; Oliveira, E. J.; Agra, M. F.; Harley, R. M.; Giulietti, A. M.; Biochem. Syst. Ecol. 2006, 34, 263.

4. Montforts, F. P.; Mueller, C. M.; Lincke, A.; Liebigs. Ann. Chem. 1990, 415.

5. Brandis, A. S.; Salomon, Y.; Scherz, A. Em Chlorophylls and Bacteriochlorophylls Biochemistry, Biophysics, Functions and Applications; Grimm, B.; Porra, R. J.; Rüdiger, W.; Scheer, H., eds.; Springer: The Netherlands, 2006, cap. 25.

6. Buchanan, M. S.; Hashimoto, T.; Asakawa, Y.; Phytochemistry 1996, 41, 1373.

7. Losev, A. P.; Kochubeeva, N. D.; Khimicheskaya Fizika 1990, 9, 616. (CAN 114:23299).

8. Watanabe, N.; Yamamoto, K.; Ihshikawa, H.; Yagi, A.; Sakata, K.; Brinen, L. S.; Clardy, J.; J. Nat. Prod. 1993, 56, 305.

9. Wegerski, C. J.; France, D.; Cornell-Kennon, S.; Crews, P.; Bioorg. Med. Chem. 2004, 12, 5631.

10. Squier, A. H.; Hodgson, D. A.; Keely, B. J.; Org. Geochem. 2002, 33, 1655.

11. Louda, J. W.; Liu, L.; Baker, E. W.; Org. Geochem. 2002, 33, 1635.

12. Ocampo, R.; Repeta, D. J.; Org. Geochem. 1999, 30, 189.

13. Demberelnyamba, D.; Ariunaa, M.; Shim, Y. K.; Int. J. Mol. Sci. 2008, $9,864$.

14. Sharma, S.; Dube, A.; Bose, B.; Gupta, P. K.; Cancer Chemother. Pharmacol. 2006, 57, 500.

15. Di Stefano, A.; Ettorre, A.; Sbrana, S.; Giovani, C.; Neri, P.; Photochem. Photobiol. 2001, 73, 290.

16. Dias, C. S.; Moura, M. D.; Cabral, A. G. S.; Mota, S. G. R.; Da-Cunha, E. V. L.; Silva, T. M. S.; Harley, A. M. G.; Barbosa-Filho, J. M.; LABCIENCIA Not. Técn. Labor. 2007, 1, 14.

17. Silva, D. A.; Silva, T. M. S.; Lins, A. C. S.; Costa, D. A.; Matias, W. N.; Cavalcante, J. M. S.; Braz-Filho, R.; Vanderlei, M. F. S.; Quim. Nova 2006, 29, 1250. 